Nicolas C. Kahn ${ }^{1,2}$, Sabine Bartel ${ }^{3}$, Andre F.S. Amaral ${ }^{4}$, Lieuwe D. Bos ${ }^{5}$, Jana De Brandt ${ }^{6}$ on behalf of the ERS Early Career Members Committee [1]

1Pneumology and Critical Care Medicine, Thoraxklinik at Heidelberg University Hospital, Heidelberg, Germany.

${ }^{2}$ Translational Lung Research Center, Member of the German Center for Lung Research, Heidelberg, Germany. ${ }^{3}$ Early Life Origins of Chronic Lung Disease, Research Center Borstel, Leibniz-Center for Medicine and Biosciences, Member of the

German Center for Lung Research (DZL), Borstel, Germany. ${ }^{4}$ Population Health and Occupational Disease, National Heart and Lung Institute, Imperial College, London, UK. ${ }^{5}$ Academic Medical Center, University of Amsterdam, Dept of Respiratory Medicine and Dept of Intensive Care, Amsterdam, The Netherlands. ${ }^{6}$ REVAL - Rehabilitation Research Center, BIOMED -

Biomedical Research Institute, Faculty of Medicine and Life Sciences, Hasselt University, Diepenbeek, Belgium.

\title{
New kids on the block in the ECMC and opportunities for early career members in 2018
}

\section{Early Career Forum}

\section{New kids on the block in the ECMC}

The Early Career Members Committee (ECMC) has two new members, who have joined since the ERS International Congress in Milan. The new committee members are Andre F.S. Amaral (Assembly 6) and Jana De Brandt (Assembly 9).

Andre F.S. Amaral is the new representative of the early career members from ERS Assembly 6 (Epidemiology and Environment). $\mathrm{He}$ is an epidemiologist working at the National Heart and Lung Institute, Imperial College London, where he is involved in large population-based studies of lung function, asthma and chronic obstructive pulmonary disease (ALEC; ECRHS; and BOLD) and a panel study of the molecular effects of shortterm exposure to air pollution on lung function (EXPOsOMICS). He is associate editor of the journals BMC Pulmonary Medicine and Frontiers in Public Health (Epidemiology), and social media editor of Occupational and Environmental Medicine.

Jana De Brandt is the new representative of the early career members from ERS Assembly
9 (Allied Respiratory Professionals). She is a human movement scientist and clinical exercise physiologist working in the Department of Rehabilitation and Physiotherapy of Hasselt University, Belgium. For her PhD project, under the supervision of Martijn Spruit, she is actively involved in research about nutritional supplementation in combination with pulmonary rehabilitation in patients with COPD, in close collaboration with Jessa Hospital (Hasselt) and Ghent University (Ghent). Within the ECMC she is responsible for the Early Career Forum articles in Breathe and the ECMC social media communication.

\section{Opportunities for early career members in 2018}

\section{The ERS competence list of early career members}

There are many opportunities for early career members in the ERS to engage in the activities of the society. However, in the past it has not been easy for early career members to make themselves
Cite as: Kahn NC, Bartel S, Amaral AFS, et al. New kids on the block in the ECMC and opportunities for early career members in 2018. Breathe 2018; 14: 55-57. 
visible to the ERS and eligible for tasks that they feel able and competent enough to complete.

In recent years, the ECMC has established a "competence list". The goal of this list was to create a tool for ERS officers to select suitable early career members and offer them participation in specific processes of the society. Since it is the declared aim of the ERS to involve more early career members in many different aspects of the society's work, the competence list has gained importance over time. Any early career member willing to take over tasks like abstract reviewing and co-chairing poster sessions for the ERS conferences or volunteering to co-author, for example, review articles in ERS journals can sign up for this list by simply contacting his or her respective assembly's early career representative.

The ECMC was certain that, despite all the communication efforts in recent years, not all early career members knew of the existence and importance of this list. Therefore, the ECMC together with the ERS office developed an item on the myERS platform where any early career member willing to commit time and effort to the ERS can sign up to the official early career member competence list.

While filling out the online form, early career members will be asked to name their fields of expertise (up to three). It is also possible to link to your published articles in the PubMed database. You will be asked to select the different (or all!) ERS tasks that you think you are most capable of completing. To date, the available activities are: 1) reviewing abstracts for ERS conferences; 2) co-chairing sessions during ERS conferences; 3) co-authoring articles for ERS journals; and 4) serving as reviewer in the evaluation process of applications submitted for ERS fellowships. To highlight early career members that have previously undertaken ERS tasks or who have won ERS poster/ abstract awards, we decided to incorporate a section where this can be mentioned, so that chances of being selected again in the future are higher.

The ERS, in general, and the ECMC, in particular, sincerely hope that with this new myERS competence list it will be easier and more transparent for any early career member in the society to have a better chance to become involved in the many tasks and activities that the ERS has to offer.

We invite you to use your myERS account to sign up for the early career members' competence list and become an active member of the society!

\section{Early career delegates session at the Lung Science Conference 2018 in Estoril, Portugal}

It is now an established tradition during the annual Lung Science Conference (LSC) to have a session dedicated to the professional needs of the early career members.
For the past 2 years, we have been able to offer the early career members comprehensive sessions focused on optimising their chances to obtain jobs and funding. This year's session will address a vital aspect of every scientist's life: maximising their publication output.

The invited panel of experts, including the new chief editor of the European Respiratory Journal, Martin Kolb, will give the audience an insight on crucial aspects of creating and submitting manuscripts. Improving the visibility of papers once accepted for publication is another aspect the session will address.

These sessions traditionally involve lively discussions between the audience and the invited panel before turning into a networking event. This year, we aimed to increase the interaction even further. As there will be a significant number of experienced editors present during the LSC, we plan to invite the audience to bring along their own manuscripts that were not as successful in the publication process as the authors had initially hoped. The editors will make themselves visible after the panel discussion, creating the opportunity for early career members to get practical advice on the best way to improve their chances of publication.

We hope this combination of lectures and hands-on experience will lead to a spike in publications among early career members after the LSC!

\section{Early career member session at the ERS International Congress in Paris 2018}

Following on from the very successful past congresses, the ERS International Congress in Paris, September 15-19, 2018, will again offer a session dedicated to supporting ERS early career members. The session, which is organised by the ECMC, will take place on Tuesday afternoon (14:45-17:00) and will be followed by a networking evening event. This year's excellent selection of speakers aims to provide important insights into the critical steps for your successful future career in respiratory research and/or medicine. The session will start with a talk about "The ingredients for a successful career in the respiratory field: what every young investigator needs to know". The talk by Maria Belvisi (Astra Zeneca and Imperial College London) will highlight the new perception of transitioning between academia and industry in the 21st century and how this has changed in recent years. The ECMC will be represented by its chair, Lieuwe Bos (University of Amsterdam), who will provide tips for early career members on how to get actively involved in the ERS. The session will be closed by a presentation from Rory Morty (Max Planck Institute Bad Nauheim) on the extensive 
ERS fellowship programme and a current ERS fellow will share his/her experiences. The ECMC is very much looking forward to meeting many early career members in this session or afterwards at the networking event, where you will be able to get in touch with us and all the speakers.

\section{Conflict of interest}

Disclosures can be found alongside this article at breathe.ersjournals.com 